\title{
Efeitos da fisioterapia aquática na deficiência visual: relato de caso
}

\author{
Effects of aquatic therapy on visual impairment: a case study
}

\author{
Matheus Marques e Marques', Jorge Cutlac Neto', Nuno Miguel \\ Lopes Oliveira ${ }^{3}$, Carla Cristina Esteves Oliveira'2, Gualberto Ruas', \\ Suraya Gomes Novais Shimano ${ }^{3}$
}

\section{RESUMO}

Objetivo. Avaliar os benefícios da fisioterapia aquática nas capacidades musculoesqueléticas e cardiorrespiratórias e no índice de qualidade de vida (QV) em um deficiente visual. Método. A amostra foi composta de um indivíduo portador de deficiência visual adquirida, de 50 anos, sem comprometimentos neuromusculoesqueléticos. Foram utilizados: a dinamometria de preensão palmar para avaliação da força muscular de membros superiores, o teste de oito repetiçôes máximas para os membros inferiores; o Banco de Wells para flexibilidade; o teste de velocidade de marcha para funcionalidade; a espirometria para força dos músculos inspiratórios e expiratórios e o questionário WHOQOL BREF para qualidade de vida. Após a construção de um protocolo de exercícios com comandos áudio-táteis, os mesmos foram aplicados por 12 semanas, duas vezes por semana, durante $60 \mathrm{minu}$ tos. Resultados. Aumento de 5,2\% na força muscular de membros superiores e $30 \%$ de membros inferiores. $\mathrm{O}$ ganho de flexibilidade foi de $100 \%$, refletindo em melhora de 25 milissegundos no teste de velocidade de marcha. A força muscular inspiratória obteve ganho de $57,1 \%$ a melhora de QV total foi de 5,7\%. Conclusáo. Um protocolo de hidrocinesioterapia adaptado a deficientes visuais foi eficaz na promoção de ganhos físico funcionais e de QV de um deficiente visual.

Unitermos. Hidroterapia, Cegueira, Exercícios Aquáticos, Qualidade de Vida

Citação. Marques MM, Cutlac-Neto J, Oliveira NML, Oliveira CCE, Ruas G, Shimano SGN. Efeitos da fisioterapia aquática na deficiência visual: relato de caso.

\begin{abstract}
Objective. This study aimed the evaluation of benefits in aquatic therapy on musculoskeletal system, cardiorespiratory capacity and life's quality index of a blind person. Method. A blind 50 years old individual participated in this study. A dynamometer was used to evaluate the handgrip strength; the Eight Repetition Maximum Test to lower limbs strength; the Wells bank to flexibility; the gait speed test to functionality; the spirometry to respiratory muscles strength; and the Whoqol-bref to quality of life. The aquatic physical therapy protocol with audio-tactile commands was applied for 12 weeks, at frequency of twice a week for 60 minutes. Results. There was an increase of $5.2 \%$ in handgrip strength and $30 \%$ at lower limbs. The flexibility improved $100 \%$, reflecting a gain of 25 milliseconds in the gait speed test. The inspiratory muscle strength increased $57.1 \%$ was obtained. The improvement of overall quality of life was $5.7 \%$. Conclusions. This study demonstrated an adapted protocol of hydrotherapy was effective in promoting physical functional gains and quality of life of an individual with visual impairment. It is suggested that further studies with a larger population so they can be statistically proven results.
\end{abstract}

Keywords. Hydrotherapy, Blind, Aquatic Exercises, Quality of Life

Citation. Marques MM, Cutlac-Neto J, Oliveira NML, Oliveira CCE, Ruas G, Shimano SGN. Effects of aquatic therapy on visual impairment: a case study.
Trabalho realizado no Instituto de Cegos do Brasil Central, Uberaba-MG e Universidade Federal do Triângulo Mineiro, Uberaba-MG, Brasil.
Endereço para correspondência Suraya GN Shimano R. Manoel Coelho, 199 ap102, bl 04 CEP 38055-600, Uberaba-MG, Brasil

E-mail: surayagnovais@gmail.com

1.Graduando do Curso de Fisioterapia da Universidade Federal do Triângulo Mineiro, Uberaba-MG, Brasil.

2.Fisioterapeuta, Mestre em Bioengenharia pela Universidade de São Paulo Campus Ribeirão Preto, Ribeirão Preto-SP, Brasil.

3.Fisioterapeuta, Doutor, Professor Adjunto do Curso de Fisioterapia da Universidade Federal do Triângulo Mineiro, Uberaba-MG, Brasil.

4.Fisioterapeuta, Doutor em Fisioterapia pela Universidade Federal de São Carlos, São Carlos-SP, Brasil.

Relato de Caso Recebido em: 26/06/14 Aceito em: 20/02/15

Conflito de interesses: nấo 


\section{INTRODUÇÃO}

A deficiência visual é um termo utilizado para se referir à perda da visão que não pode ser reparada com lentes de correção. É considerado portador de cegueira, segundo a Organização Mundial de Saúde (OMS), o indivíduo com acuidade visual igual ou menor que 3/60 $(0,05)$ no olho de melhor visão, com a melhor correção óptica, até ausência de percepção de luz, ou perda de campo visual no olho de melhor visão com melhor correção possível. A definição de visão subnormal ou baixa visão corresponde à acuidade visual igual ou menor que $6 / 18(0,3)$ e igual ou maior do que $3 / 60(0,05)$ no olho de melhor visão após correçãoo ${ }^{1,2}$.

O controle do equilíbrio postural é realizado pela associação dos sistemas proprioceptivo, vestibular e visual, cujas informaçóes são apresentadas e processadas pelo sistema nervoso central (SNC), que emite resposta, pelas vias eferentes, para a contração dos músculos antigravitacionais, mantendo a unificaçáo deste sistema ${ }^{3}$. O sistema somatossensorial/proprioceptivo, sensível a movimentos rápidos, fornece informaçóes sobre a localização do corpo uma vez que ajuda manter o alinhamento dos segmentos corporais, contribuindo para a manutenção do equilíbrio ${ }^{4}$. O sistema vestibular permite ao indivíduo se localizar no espaço, principalmente com relação à posição de cabeça. Por fim, o sistema visual atua regulando a posição e o movimento da cabeça em relação aos objetos do meio. As informaçóes sensoriais aferem ao SNC que irá detectar se as estruturas otolíticas, que detectam a aceleração linear, estão respondendo corretamente à adaptação em relação à gravidade e ao movimento da cabeça ${ }^{3}$. Como esses sistemas atuam juntos, uma vez que algum deles se apresenta comprometido, as alterações acontecem em cascata e o processo final se encontrará falho. Desta forma, indivíduos com deficiência visual têm mais propensão a apresentarem grandes alterações de postura e equilíbrio, o que pode aumentar o risco de queda 5 . Em um mundo visual, estas disfunçóes provocam um comportamento de reclusão e sedentarismo que irão culminar em diminuição de impulsos provenientes da ação muscular e posição articular, com um quadro clínico de perda de força e flexibilidade musculares, falta de condicionamento cardiorrespiratório, exclusão social e diminuição de qualidade de vida.
Em uma abordagem interdisciplinar e com uma visão de funcionalidade, a intervenção fisioterapêutica, que objetiva a prevenção de disfunçóes e promoção da saúde, além de reabilitação os distúrbios e complicaçôes físico funcionais apresentam uma variedade de métodos e técnicas para romper esta situação de inércia e propiciar ao deficiente visual melhores condiçóes de vida e saúde ${ }^{6}$. A fisioterapia aquática é um método que utiliza os efeitos físicos, fisiológicos e terapêuticos sobrevindos da atividade física em imersão para prevenção e reabilitação de alteraçôes físico funcionais. No ambiente aquático são possíveis exercícios lúdicos e tridimensionais, sendo este o meio ideal para realização de atividades físicas, por ser seguro e eficaz'.

Com a observação de várias evidências de ganhos físico funcionais de pacientes com diversas disfunçóes ${ }^{8}$, foi levantada a hipótese de que um programa de exercícios aquáticos desenvolvido especificamente para deficientes visuais pudesse melhorar as condiçóes físicas e a qualidade de vida dos mesmos. Assim, este trabalho objetivou demonstrar os benefícios da fisioterapia aquática nas capacidades musculoesqueléticas e cardiorrespiratórias e no índice de qualidade de vida em um deficiente visual.

\section{MÉTODO}

\section{Amostra}

Trata-se de um relato de caso. $\mathrm{O}$ atendimento foi realizado no Instituto de Cegos do Brasil Central (ICBC) na cidade de Uberaba, Minas Gerais, obedecendo aos princípios éticos para pesquisa envolvendo seres humanos, Com aprovação pelo Comitê de Ética em Pesquisa da Universidade Federal do Triângulo Mineiro-UFTM, sob parecer nº 1965/2011. O paciente, depois de ter tido todas as suas dúvidas sobre o estudo esclarecidas pelos pesquisadores e tendo concordado em participar da proposta de intervenção terapêutica, assinou o Termo de Consentimento Livre e Esclarecido.

Assim, a amostra foi composta por um paciente, 50 anos, com cegueira adquirida por retinose pigmentar há 16 anos. Este possui o segundo grau completo, sendo que seu último trabalho, no período de 12 meses, foi no cargo de operador de máquina de injeção de plástico até 
receber o diagnóstico acima citado, quando foi afastado e aposentado, e passou a receber benefícios governamentais no valor de aproximadamente dois salários mínimos, que é a principal fonte de renda da sua família composta de quatro pessoas. Com relação a hábitos de prática de atividades física, é sedentário. No ICBC, além da fisioterapia aquática o paciente tem acesso a atendimentos interdisciplinares de saúde, contando com o acompanhamento preventivo e terapêutico de nutricionista, terapeuta ocupacional, assistente social e psicólogo e multidisciplinar na área educacional com processos pedagógicos que visam a inclusão social. Durante o período que o paciente participou do estudo, este não necessitou de atendimentos extra, exceto a fisioterapia aquática.

A terminologia retinose pigmentar é utilizada quando ocorrem algumas disfunçóes progressivas da retina, seja ela individualizada ou dentro de algum distúrbio, bem como a atrofia do tecido e degeneração celular?

\section{Procedimento}

As sessóes de fisioterapia aquática foram realizadas em piscina terapêutica, adaptada para deficientes visuais, com temperatura média de $32^{\circ}$, com $12,50 \mathrm{~m}$ de largura, $25 \mathrm{~m}$ de comprimento de variação de profundidade de $1,25 \mathrm{~m}$ a $1 \mathrm{~m}$. Foram realizadas 24 sessóes, com duração de 50 minutos cada, e frequência de duas vezes por semana com intervalos de $48 \mathrm{~h}$ pelo menos. O paciente foi atendido através de um protocolo de exercícios adaptados para deficientes visuais.

A intervenção foi determinada após avaliação das condiçóes físico funcionais do paciente cujos métodos serão descritos a seguir e constou de um protocolo de exercícios já utilizados em hidroterapia, porém com adaptaçôes específicas para deficientes visuais, com comandos verbais e táteis para ensino de cada exercício. Com o passar do tempo, adaptação do paciente aos exercícios e ganho de consciência corporal, este foi adquirindo independência para realizar os exercícios sem a necessidade dos comandos visuais e táteis, necessitando somente de correçóes táteis de posicionamentos e angulaçôes. A sessão de fisioterapia aquática consistia em $10 \mathrm{~min}$ de aquecimento, 30min de fortalecimento, alongamento e condicionamento cardiorrespiratório e $10 \mathrm{~min}$ de relaxamento. A realização de cada exercício permitia treino e equilíbrio e propriocepção. As séries e repetiçóes foram sendo acrescidas de acordo com a adaptação do paciente.

Os materiais utilizados para a realização dos exercícios foram flutuadores cervicais e lombares, halteres, palmares, espaguete, bastão e prancha de natação.

A avaliação funcional pré e pós-intervenção utilizando os seguintes instrumentos: o peso corporal foi mensurado em uma balança (Scale Plus Body Fat Monitor With Body Wate, capacidade de $150 \mathrm{~kg}$, precisáo de $0,1 \mathrm{~kg}$ ), com o paciente sem calçados e com roupa de banho.

A altura foi medida com um estadiômetro próprio da balança com precisão de $1 \mathrm{~mm}$ utilizando o método da estatura alongada. A equação IMC=peso/altura ${ }^{2}$ foi utilizada para a determinaçáo do índice de massa corpórea $(\mathrm{IMC})^{10}$.

A flexibilidade foi avaliada utilizando o banco de Wells (Instant Flex, Sanny ${ }^{\oplus}$, Brasil). Para realização do teste, o paciente sentou-se em frente à caixa, apoiou os pés no local indicado e manteve os joelhos estendidos. Foi solicitado que este levasse os braços estendidos à frente à régua localizada no centro do banco, sem flexionar os joelhos. O resultado escolhido foi o melhor de três tentativas em um intervalo de 30seg.

A força de preensão palmar foi determinada pelo dinamômetro hidráulico manual, modelo NC701/42-North Cost $^{\mathrm{TM}}$ seguindo as recomendações da American Society of Hand Therapysts (ASHT) ${ }^{11}$ onde o paciente permanece sentado com os dois pés apoiados no chão, ombro aduzido, cotovelo fletido a $90^{\circ}$, antebraço em posição neutra, com o polegar apontado para cima e punho em posição confortável entre 0 e $30^{\circ}$ de extensão, com ajuste à segunda posição de manopla. $\mathrm{O}$ examinador deu o comando verbal em volume alto para o paciente realizar máxima força de preensão no dinamômetro com a mão dominante. Foram feitas três medidas e utilizado o valor médio entre elas. A avaliação da força muscular de membro inferior foi realizada por meio do teste de força de oito repetições máximas (8RM) utilizando caneleiras de $0,5 \mathrm{~kg}$ à $5 \mathrm{~kg}$.

Para avaliação da marcha foi utilizada a última das três etapas da versão brasileira da Short Physical Performance Battey $(\mathrm{SPPB})^{12}$.

Para avaliação da força muscular respiratória foi utilizada a manovacuometria (aparelho, marca, unidade 
expiratória máxima (PEmáx) e pressão inspiratória máxima (PImáx). As capacidades e volumes respiratórios foram mensurados através da espirometria (Master Scope da Jäeger ${ }^{\circledR}$, Wuerzburg, Alemanha), considerando os valores das variáveis: capacidade vital forçada (CVF), volume expiratório forçado no primeiro segundo (VEF1) e a relação entre estas.

A avaliação da qualidade de vida seguiu duas etapas. A primeira que constou da avaliação cognitiva por meio do Mini Exame do Estado Mental (MEEM), sendo utilizada a versão brasileira ${ }^{13}$ com adaptação do item de habilidade construtiva de desenho de um pentágono para a montagem da figura geométrica com mini bastóes de madeira, para permitir a execução por um deficiente visual. Para a segunda etapa foi utilizado o questionário de avaliação de qualidade de vida preconizado pela Organização Mundial de Saúde WHOQOL-BREF, validado no Brasil $^{13}$, na forma lida, composto por quatro domínios: físico, psicológico, social e ambiental.

Como este é o estudo de caso único, a apresentação dos resultados foi descritiva e os resultados organizados em valores absolutos (medidas antropométricas, força muscular respiratória e esquelética e flexibilidade) e percentuais (espirometria e qualidade de vida). Os ganhos obtidos foram apresentados e discutidos em valores percentuais.

\section{RESULTADOS}

Os resultados das modificaçôes físicofuncionais promovidas pela intervenção em piscina terapêutica estão apresentados na Tabela 1 . Na avaliação da qualidade de vida houve aumento nos domínios físico, ambiental e psicológico o que por sua vez refletiu na qualidade de vida (Figura 1).

\section{DISCUSSÃo}

Observando os resultados apresentados, pode-se verificar que a fisioterapia aquática proporcionou neste indivíduo ganhos físicos evidentes tanto na melhora cardiorrespiratória quanto musculoesquelética. Estes benefícios propiciaram um ganho funcional que foi confirmado pela diminuição do tempo no teste de velocidade de mar- cha. Também refletiram em melhora na qualidade de vida deste indivíduo com deficiência visual.

Nos deficientes visuais totais ou parciais há déficits funcionais que fazem com que o corpo do indivíduo perca a sua estabilidade e seu equilíbrio ${ }^{3}$. Neste âmbito, o controle postural é um dos mais afetados, fazendo com que o indivíduo portador de deficiência visual tenha de se adaptar a sua nova condição e às limitações diárias. Tais restriçóes irão influenciar tanto suas atividades de vida diária quanto as denominadas atividades instrumentais de vida diária, ocasionando sérias dependências para realização de trabalho e lazer ${ }^{14}$. Este "efeito dominó" irá intervir diretamente na qualidade de vida destes indivíduos ${ }^{15}$.

Visando restabelecer a função biopsicossocial destes indivíduos com necessidades especiais, a fisioterapia intervém com inúmeros métodos de re/habilitação, incluindo a fisioterapia aquática, que de forma segura e prazerosa promove ganhos globais significativos.

Neste estudo, os efeitos benéficos da realização de exercícios aquáticos terapêuticos puderam ser observados no acompanhamento longitudinal por 12 semanas do deficiente visual que era frequentador assíduo do ICBC

Tabela 1. Variáveis antropométricas, espirométricas, força muscular respiratória e esquelética.

\begin{tabular}{|c|c|c|c|}
\hline \multicolumn{2}{|c|}{ Variáveis } & \multirow{2}{*}{$\frac{\text { Avaliação }}{52}$} & \multirow{2}{*}{$\begin{array}{c}\text { Reavaliaçáo } \\
51\end{array}$} \\
\hline Antropométricas & $\begin{array}{l}\text { Massa corporal } \\
\qquad(\mathrm{Kg})\end{array}$ & & \\
\hline & Estatura $(\mathrm{cm})$ & 166 & 166 \\
\hline & IMC $\left(\mathrm{kg} / \mathrm{m}^{2}\right)$ & 18 & 18 \\
\hline \multirow[t]{4}{*}{ Espirométricas } & CVF (\% prev) & 74 & 75 \\
\hline & VEF1 (\% prev) & 85 & 86 \\
\hline & VEF1/CVF (\%) & 113 & 114 \\
\hline & VVM (\% prev) & 90 & 110 \\
\hline \multirow[t]{2}{*}{$\begin{array}{l}\text { Força muscular } \\
\text { respiratória }\end{array}$} & $\begin{array}{l}\text { PImáx } \\
(\mathrm{cmH} 2 \mathrm{O})\end{array}$ & 70 & 110 \\
\hline & $\begin{array}{c}\text { PEmáx } \\
(\mathrm{cmH} 2 \mathrm{O})\end{array}$ & 75 & 90 \\
\hline \multirow{2}{*}{$\begin{array}{c}\text { Força muscular } \\
\text { esquelética }\end{array}$} & Dinamômetria & 38 & 40 \\
\hline & $8 \mathrm{RM}$ & 10 & 13 \\
\hline Flexibilidade & Banco de Wells & 7 & 14 \\
\hline $\begin{array}{c}\text { Velocidade de } \\
\text { marcha }\end{array}$ & 4 metros & 445 & 420 \\
\hline
\end{tabular}

IMC = Índice de Massa Corporal; CVF = Capacidade Vital Forçada; VEF1 = Volume Expiratório Forçado no primeiro segundo; VEF1/CVF = Índice de Tiffneau; VVM = Ventilação Voluntária Máxima; PImáx = pressão inspiratória máxima; PEmáx = pressão expiratória máxima; $\mathrm{RM}$ = repetiçōes máximas. 


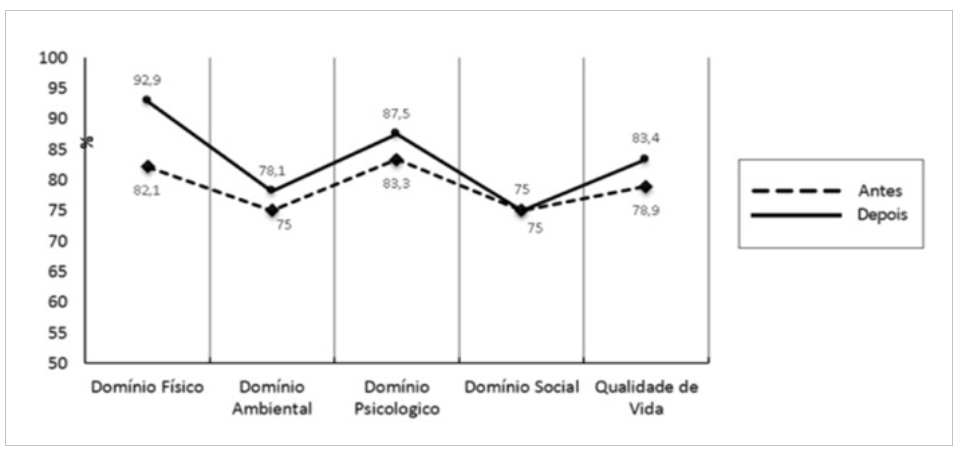

e que aderiu totalmente ao programa de exercícios aquáticos proposto.

Houve manutenção das características antropométricas, visto que o indivíduo não apresentava em sua avaliação inicial índices de sobrepeso ou obesidade segundo a Organização Mundial de Saúde ${ }^{16}$.

O ganho expressivo de flexibilidade apontado nos resultados das avaliaçóes (100\%) demonstra que o remodelamento dos tecidos conjuntivo, muscular e tendinoso, assim como a adaptação nas proteínas miocontráteis dos sarcômeros foram alcançados pelos exercícios propostos no protocolo e, provavelmente, acelerados pelos efeitos fisiológicos da imersão ${ }^{17}$.

O ganho de força e flexibilidade esta intimamente ligada ao ganho proprioceptivo o que reflete em ganho de equilíbrio estático e dinâmico. Desta forma, indivíduos com deficiência visual, que estão privados deste tipo de informação sensorial e, consequentemente estão mais instáveis, podem ter maior facilidade em manutenção da postura ereta correta ${ }^{18}$. Nesse caso, a supressão visual e a instabilidade postural são compensadas pela estimulação de outras áreas sensoriais ${ }^{19}$.

Visando os benefícios supracitados, a escolha de um protocolo de fisioterapia aquática objetivou aprimorar a recepção de informaçóes sensoriais para melhora postural e manutenção de equilíbrio, assim possibilitando a interação de diferentes sistemas sensoriais. Os resultados deste tipo de treinamento se refletem em melhora do equilíbrio e propriocepção em pisos estáveis e instáveis, possibilitando reaçôes positivas em momentos de instabilidade postural ${ }^{20}$, prevenindo quedas.
A fisioterapia aquática, embora enfatize o ganho de força, o protocolo proposto envolvia exercícios de resistência muscular que, neste período, não promoveram hipertrofia muscular. Estes achados corroboram com os apresentados por diversos autores no mesmo período de tempo ${ }^{21}$.

Para avaliação da força muscular foi utilizado dos métodos largamente discutidos na literatura, a dinamometria de preensão palmar, e o teste de 8RM. Em ambas as análises foram obtidas ganhos, havendo melhora de 5,25 e 30\% respectivamente. Os ganhos obtidos na preensão palmar são de especial importância, pois são preditores de ganho de força muscular global ${ }^{22}$. Essa resposta fisiológica é explicada por diversos autores ${ }^{23,24}$ que afirmam que as propriedades físicas da água, quando associadas à cinesioterapia, podem promover uma melhora da força muscular e da amplitude de movimento articular, favorecendo um ganho funcional. Considerando que o aumento fisiológico de força de preensão ocorre até os 39 anos e, posteriormente, há uma diminuição gradual, o ganho de força obtido neste estudo revela uma melhora considerável neste parâmetro que deveria estar em declínio $^{22}$. Provavelmente com a continuidade dos treinamentos, incrementando a carga, que na hidrocinesioterapia se traduz em aumento da velocidade movimento, da turbulência e do tamanho de flutuadores, pode haver mais ganho de força.

De acordo com o teste de velocidade de marcha, o tempo foi diminuído, demonstrando a eficácia do tratamento nesta variável. Essa melhora pode ser explicada pelos estímulos ao equilíbrio somados aos ganhos físicos. 
A função de marcha revela que a melhora em parâmetros físicos reflete em ganhos funcionais.

Em relação às variáveis espirométricas todas obtiveram ganho, porém a Ventilaçâo Voluntária Máxima (VVM) foi a que apresentou maior significância, de aproximadamente $22 \%$. A força muscular respiratória obteve melhora tanto na pressão PImax quanto na PEmax, sendo aproximadamente $57 \%$ e $20 \%$ respectivamente.

O trabalho respiratório aumenta durante a imersão com profundidade mínima do processo xifóide, de forma que tanto a mecânica quanto a função pulmonares sofrem alteraçóes. Isto ocorre devido à pressão hidrostática, que irá intervir diretamente sobre esse sistema, com aumento do volume central de sangue (por compressão de vasos sanguíneos e linfáticos de membros superiores e inferiores) e compressão da caixa torácica e o abdome, resultando em diminuição da complacência e das capacidades pulmonares. É necessário, portanto um aumento do trabalho da musculatura inspiratória, para manter os volumes pulmonares. Consequentemente o diafragma, principal músculo da respiração, é solicitado constantemente durante a realização de exercícios aquáticos, com sobrecarga extra, tendo ganho de força muscular considerável ${ }^{7,23-26}$.

$\mathrm{Na}$ reavaliação pode-se perceber que houve uma melhora significativa da qualidade vida, sendo os domínios que obtiveram expressiva melhora o físico, o ambiental e o psicológico, enquanto o domínio social manteve seu índice inalterado

Os ganhos físico funcionais obtidos durante a realização de exercícios de um protocolo adaptado permitiram não somente incremento de capacidades musculoesqueléticas e cardiorrespiratórias. Também possibilitaram uma independência funcional e convívio social que podem ter influenciado a auto-confiança, confirmada pelo aumento significativo do domínio psicológico. A realização regular de exercícios irá incrementar tanto a condição física quanto influenciar no bem estar modificando fatores emocionais e sociais influenciando diretamente na melhora da qualidade de vida, o que pode ser comprovado no presente estudo ${ }^{27}$. Dessa forma se percebe que a terapia aquática deve ir de encontro com a necessidade de cada paciente, sendo uma terapia humanizada e que visa à readaptação do deficiente visual de forma holística ${ }^{27}$.
Uma limitação do presente estudo foi a amostra de apenas um deficiente visual. Porém os resultados positivos do presente estudo demonstram que ganhos similares podem ser obtidos por outros deficientes visuais que participarem efetivamente de um programa de exercícios adaptados.

\section{CONCLUSÃO}

O presente estudo demonstra que um protocolo de hidrocinesioterapia baseado em comandos áudio-táteis foi eficaz na promoção de ganhos físico funcionais e de qualidade de vida de um indivíduo com deficiência visual. Sugere-se a realização de mais estudos com uma população maior para que possam ser comprovados estatisticamente os resultados.

\section{AGRADECIMENTO}

Ao Instituto de Cegos do Brasil Central e ao voluntário, portador de deficiência visual, que prontamente colaborou com a realização do estudo.

\section{REFERÊNCIAS}

1.Nakamani CR. O que é considerado baixa visão e cegueira dos pontos de vista oftalmológico, educacional e legal. In: Rodrigues MLV. Saúde Ocular e Prevençấo da Cegueira. Rio de Janeiro: Cultura Médica, 2009, p.18-23.

2.Rinaldo GRC. Funçôes Visuais: Acuidade Visual. In: Schor PS, Uras R, Veitzman S. Óptica, Refração e Visão Subnormal. Rio Janeiro: Cultura Médica, 2008, p.423-8.

3.Baraúna MA, Barbosa CRM, Canto RST, Silva RAV, Silva CDC, Baraúna KNP. Estudo do equilíbrio estático de idosos e sua correlação com quedas. Fisioter Bras 2004;5:136-41.

4.Aires M. Fisiologia. 3a edição. Rio de Janeiro: G Koogan, 2008, 1232p.

5.Bittar RSM, Pedallini MEB, Bottino MA, Formigoni LG. Síndrome do desequilíbrio no idoso. Pró-Fono 2002;14:119-28.

6.Conselho Federal de Fisioterapia e Terapia Ocupacional. Definição de Fisioterapia (Endereço na Internet). Brasilia: COFFITO (acessado em 06/2014). Disponível em: http://www.coffito.org.br

7.Routi RG, Morris DM, Cole AJ. Reabilitação aquática. São Paulo: Manole, 2000, 466p.

8.Biasoli MC, Machado CMC. Hidroterapia: técnicas e aplicabilidades nas funçōes reumatológicas. Temas Reum Clin 2006;7:78-87.

9.Tayah D, Angelucci RI, Sampaio P, Rehder JRCL. Retinose Pigmentar. Arq Med ABC 2004;29:82-6.

10.Norton KOT. Antropométrica. Porto Alegre: Artmed, 2005, 398p.

11.Abdalla IM, Brandão MCF. Força de preensão palmar. In: Manual de recomendaçôes para avaliação do membro superior. 2. ed. São Paulo: Sociedade Brasileira de Terapeutas da Mão, 2005, p.263-84. 
12.Nakano MM. Versão Brasileira da Short Physical Performance Battery SPPB: Adaptação Cultural e Estudo da Confiabilidade (Dissertação). Campinas: Faculdade de Educação, Universidade Estadual de Campinas - UNICAMP, 2007, 181p.

13.Fleck MPA, Lousada S, Xavie M, Chachamovich E, Vieria G, Santos L, et al. Aplicação da versão em português do instrumento abreviado de avaliação da qualidade de vida "Whoqol-bref". Rev Saude Pub 2000;2:178-83. dx.doi. org/10.1590/S0034-89102000000200012

14.Bellew JW, Panwitz BL, Peterson L, Brock MC, Olson KE, Staples WH. Effect of acute fatigue of the hip abductors on control of balance in young and older women. Arch Phys Med Rehab 2009;90:1170-5. dx.doi.org/10.1016/j. apmr.2009.01.025

15. Oliveira DN, Barreto RR. Avaliação do equilíbrio estático em deficientes visuais adquiridos. Rev Neurocienc 2005;13:122-7.

16.WHO. Diet, nutrition and the prevention of chronic diseases. World Health Organ Tech Rep Ser 2003; 916:1-149.

17.Signori LU, Voloski FRS, Kerkhoff AC, Brignoni L, Plentz RDM. Efeito de agentes térmicos aplicados previamente a um programa de alongamentos na flexibilidade dos músculos isquiotibiais encurtados. Rev Bras Med Esp 2008;14: 328-31.

18.Nakata H, Yabe K. Automatic postural response systems in individuals with congenital total blindness. Gait Post 2001;14:36-43. dx.doi.org/10.1016/ S0966-6362(00)00100-4
19.Easton RD, Greene AJ, Dizio P, Lackner JR. Auditory cues for orientation and postural control in sighted and congenitally blind people. Exp Brain Res 1998;118:541-50.

20.Resende S, Rassi C, Viana F. Efeitos da hidroterapia na recuperaçáo do equilíbrio e prevenção de quedas em idosas. Rev Bras Fisioter 2008;12:57-63. dx.doi.org/10.1590/S1413-35552008000100011

21.Komi PV. Força e potência no esporte. Porto Alegre: Artmed, 2008, 472p. 22.Caporrino FA, Faloppa F, Santos JB, Réssio C, Soares FH, Nakashima LR, et al. Estudo populacional da força de preensão palmar com dinamômetro Jamar. Rev Bras Ortop 1998;33:150-4.

23.Becker BE, Cole AJ. Terapia Aquática Moderna. São Paulo: Manole, 2000, 202p.

24.Campion MR. Hidroterapia: princípios e prática. São Paulo: Manole, 2000, 332p.

25.Kurabayashi H, Machida IH, Akida T, Kubota K. Comparison of three protocols for breathing exercises during immersion in $38^{\circ} \mathrm{C}$ water for chronic obstructive pulmonary disease. Am J Phys Med Rehabil 1998;77:145-8.

26.Agostoni E, Gurtner G, Torri G, Rahn H. Respiratory mechanics during submersion and negative-pressure breathing. J Appl Physiol 1986;21:251-8. 27.Iversen MD, Fossel AH, Ayers K, Palmsten A, Wang HW, Daltroy LH. Predictors of exercise behavior in patients with rheumatoid arthritis 6 months following a visit with their rheumatologist. Phys Therp 2004;84:706-16. 